use of different calibrators and by the fact that drug tolerance differs among assays ranging from extreme drug sensitive over various forms of drug tolerant to drug resistant anti-drug antibody asssays. The clinical relevance of the different type of anti-drug antibody assays remains to be proven.

Combining therapeutic drug concentrations and anti-drug antibody concentrations with relevant patient, disease and drug information will lead to optimal dosing of patients aiming at optimal clinical, biochemical and endoscopic outcomes.

Disclosure of Interest: A. Gils Grant/research support from: IIR grants from pfizer, Speakers bureau: speakers fee by Pfizer, MSD, Abbvie, Takeda, JnJ

DOI: 10.1136/annrheumdis-2017-eular.7210

\section{SP0083 AS A RHEUMATOLOGIST, DOES IT HAVE ANY CONSEQUENCE IN MY DAILY PRACTICE?}

J.W. Bijlsma. Rheumatology \& Clinical Immunology, UMCU, Utrecht, UTRECHT, Netherlands

It is nearly inevitable that when we administer foreign (even humanised) proteins intravenously or subcutaneously to a person, that said person will develop antibodies to that (foreign) protein. This happens to most of our patients when we administer biologicals; depending on the sensitivity of our methods, we can measure these anti-bodies easily or not at all. These antibodies start becoming a problem when they are actually binding the administered biological, thus making the active drug less available for its targeted function. We can evaluate this by measuring the actual drug-level, so called trough level. Numerous reports have been published, showing that there is indeed a negative correlation between e.g. anti-tumor necrosis factor (TNF) drug antibodies and the efficacy of anti TNF in the treatment of RA. It has also been shown that adding methotrexate (MTX) to the anti-TNF treatment improves its efficacy and reduces the level of anti-drug antibodies. Probably only $10 \mathrm{mg}$ MTX weekly would be enough to obtain this effect.

So what do I do as a clinician when I observe that a patient, who originally did very well, loses response to her biological? Do I measure possible anti-drug antibodies? No, the consequences are zero: When the patient is not responding to the given drug anymore, I need to adapt the treatment; the drug she is using is not effective anymore, so we should change. Would the presence of anti-drug antibodies influence my decision? No, there is no cross-reactivity to other biologicals (even from the same class of action), except to its biosimilar (underscoring that it is a real biosimilar!). In case there is doubt whether a patient is actually using the biological we could better measure the drug-trough level; but -in my practice- this question seldom arises in patients with active arthritis, being treated with a biological.

Measuring drug-trough levels is a completely other item, and perhaps more relevant. Biologicals are in general given in a standard fixed dosage, while there are clear differences in patients characteristics, that could influence bioavailability of the biological. In addition when the disease is more active, it could be that more biological is needed to temper the inflammation compared to low disease activity, where perhaps a lower dosage would be more than effective. To guide physician and patient in personalizing and optimizing treatment with biologicals measuring drug-trough levels might be helpful. Different studies have been performed trying to use through level of the drug in adapting the dosage, and even in predicting possibility to stop the drug treatment. This area is still being evaluated and it is too early to make firm statements, but with a look at cost-effectiveness this will certainly become relevant.

Coming back to the original question: do I use anti-drug antibodies in my daily practice to guide treatment: no, it doesn't influence my decisions. Will I use in the future drug trough levels to guide treatment decisions: this could well be, but it is too early to make a final decision yet.

Disclosure of Interest: None declared

DOI: 10.1136/annrheumdis-2017-eular.7118

\section{THURSDAY, 15 JUNE 2017 \\ Which target / outcome is more relevant in the management of SLE?}

\section{SP0084 BIOLOGICAL TARGETS IN SLE}

C. Chizzolini on behalf of Swiss SLE Cohort Study and PRECISESADS consortium. Immunology \& Allergy, Geneva University Hospital, Geneva, Switzerland

SLE is a prototypical condition characterized by the complete subversion of immunological tolerance and the generation of autoantibodies directed against a wide array of ubiquitous and tissue-specific antigens. This is possible because the joint dysregulation of the innate and adaptive arms of the immune system; which results from multiple gene polymorphisms, each contributing marginally, distinct epigenetic regulation, alteration of the threshold of activation for $T$ and $B$ cells, enhanced responses of antigen-presenting cells resulting from the altered disposal of apoptotic cells, as well as dysregulation of cytokine circuitries including regulatory networks.

Pathogenic mechanisms resulting in clinically overt SLE very likely are het- erogeneous among individuals. Thus, the identification of biological targets in SLE goes also with the identification of selected modules of gene activation in distinct individuals. Very strong signals indicate that type I interferon (IFN) may contribute to autoimmunity in a large proportion of SLE individuals and therapeutic trials targeting IFN signaling suggest the clinical relevance of this mediator. B cells/plasmablasts are also relevant and obvious targets. Refinements in our understanding in B cell sub setting and/or the timing in disease development in which they play a relevant role should result in defining the appropriate targets specific to this cell lineage. Gene modules activated during flares suggest that neutrophils in a subset of individuals may also be relevant targets. Cytokine affecting $\mathrm{T}$ cell differentiation, in particular $\mathrm{T}$ follicular helper cells, represent additional relevant targets.

Within the last several years a number of novel biological targets have been identified in SLE. However, a single biological agent has been approved for SLE treatment in the last five decades. This underlies the difficulties encountered when translating validated targets in efficacious therapeutic agents. This stress the need for careful preclinical evaluation. It further emphasizes the need of small phase II clinical trials based on stringent inclusion criteria aiming at precisely identifying individual groups more likely to respond to validate the target. Current progress made in the identification of molecular signatures in individuals with SLE will offer the tools for the requested accurate selection.

Disclosure of Interest: C. Chizzolini Grant/research support from: Unrestricted reserach grant form GSK

DOI: 10.1136/annrheumdis-2017-eular.7228

\section{SP0085 PATIENT REPORTED OUTCOMES}

M. Schneider. Policlinic \& Hiller Research Unit for Rheumatology, HHUD, Duesseldorf, Germany

In SLE as in other rheumatic diseases, the most relevant target of intervention should be a status with controlled disease process assuring no further accrual of damage. If actual expert discussions like DORIS define the frame of such a status, clinical activity measured by a validated lupus disease activity instrument, serologic activity and therapy - because of harm - are the dimensions of remission with its duration as additional factor for outcome. Patient reported outcomes (PROs) were not included. Otherwise, if payers and reimbursement system decide about relevance, patient outcomes are clear of highest importance as target.

Looking on the evidence of PROs for outcome in SLE, PROs were never used as primary endpoint in clinical trials. In RCTs, PROs were often collected and mostly explorative analysed. There is no evidence that PROs can validly define the above described status of controlled disease. But from systematic analyses in RA, we know that pure PRO like VAS of general health status and semi PRO like tender joints are at least as relevant as more "objective" criteria like swollen joints or CRP as clearly exhibited by the ACR/Eular remission criteria for RA. The challenge in SLE is that the discrepancies between patients' and physicians' perception and perspectives are even more distinct than in RA. Sometimes, there is the expression that physicians and patients are describing different diseases. The burden of illness in lupus is better defined by pain than by organ manifestations; the overall survival in SLE is more related to lupus nephritis than to fatigue. It is obvious that physicians should analyse the actual clinical symptoms and integrate the future consequences of their actual management in their decision, and patients are more focused on release of their actual burden. Until today, these different and divers perspectives are no integrated, neither in RCTs nor in daily care. But such integration is mandatory, because no side imagines the complete picture of lupus, which may also produce to the poor results of clinical trials. In routine care, this behaviour causes frustration and mental distress, optimal results are prohibited.

So, the answer to what is more relevant in the management of SLE patients clinical targets, biological targets or PROs - is the integration of all important aspects of lupus. This implies more than the statistical evaluation of the best items of all three aspects, it is the active involvement of patients in their care: patient empowerment in SLE, a fruitful process, in which both sides have to learn a lot from and about each other.

Disclosure of Interest: M. Schneider Shareholder of: no, Grant/research support from: GSK; UCB, Consultant for: Astra-Zeneca; GSK; Lilly; Roche; Suppremol; UCB, Employee of: no, Paid instructor for: no, Speakers bureau: Astra-Zeneca; GSK; Roche; Pfizer; UCB

DOI: 10.1136/annrheumdis-2017-eular.7248

\section{THURSDAY, 15 JUNE 2017}

\section{Joint EULAR - EFIS session: Tilting the balance: from disease to tolerance induction}

\section{SP0086 PATHOGENIC MEMORY CELLS: ROAD BLOCKS TO TOLERANCE INDUCTION?}

H.-D. Chang. German Rheumatism Research Center Berlin, Berlin, Germany

While conventional state-of-the-art immunosuppression can lead to significant 